\title{
Implantação da iniciativa Hospital Amigo da Criança em um Hospital Universitário ${ }^{1}$
}

\section{Implantation of the Baby Friendly Hospital initiative at a University Hospital}

\author{
Marli T. Oliveira Vannuchi²; Andreza D. D. Sentone ${ }^{3}$; \\ Carlos Augusto Monteiro ${ }^{4}$ Marina Ferreira Réa ${ }^{5}$
}

\section{Resumo}

\begin{abstract}
Este artigo tem como objetivos descrever o processo de implantação da Iniciativa Hospital Amigo da Criança (IHAC) no Hospital Universitário Regional do Norte do Paraná (HURNP) e destacar as dificuldades e benefícios advindos dessa implantação. A metodologia empregada foi a utilização de inquéritos, com base nos "Dez Passos", realizados antes e após a implantação do IHAC no HURNP. O inquérito realizado após a implantação, revelou o cumprimento dos "Dez Passos" e o aumento significativo dos índices de aleitamento exclusivo (de 6,7\% para 95,0\%). A implantação da IHAC no HURNP, por ser um hospital universitário, representou um grande desafio para a equipe, porém levou a mudança no comportamento e práticas relativas à amamentação, resultando no aumento dos índices de aleitamento materno e aleitamento materno exclusivo dos bebês que nasceram neste hospital.
\end{abstract}

Palavras-chave: Criança, aleitamento materno, desmame precoce.

\begin{abstract}
The goals of this article are to describe the "Baby Friendly Hospital Initiative" implantation at the University Hospital of Northern Paraná, situated in Londrina, and point out the difficulties and advantages of this process. The methodology consisted of two inquiries based on the "Tem steps for the success of breast feeding" which were carried out before and after the "Baby Friendly Hospital Initiative" implantation at the hospital. The inquiry carried out after the implantation at the hospital showed the accomplishment of the ten steps and the significant increase of the levels of exclusive breast-feeding (6,7\% to 95\%). Considering that this work was developed at a school hospital, it was a great challenge for the health professional group, showing changes in behavior and breast-feeding practices, therefore, resulting in an important increase of breast-feeding levels.
\end{abstract}

Key-words: Baby, breast-feeding, early wean.

\footnotetext{
${ }^{1}$ Parte da tese de Doutorado apresentada à Faculdade de Saúde Pública da USP. Trabalho realizado no Hospital Universitário Regional do Norte do Paraná, Londrina.

2 Professora Doutora do Departamento de Enfermagem da UEL.. Membro do Banco de Leite do HURNP e da Rede IBFAN. email:vannuchi@sercomtel.com.br

3 Enfermeira Chefe da Divisão Materno-Infantil do HURNP.

${ }^{4}$ Professora Doutora do Instituto de Saúde de São Paulo.

5 Professor Titular do Departamento de Nutrição da Faculdade de Saúde Pública da USP.
} 


\section{Introdução}

A Iniciativa Hospital Amigo da Criança (IHAC) é uma estratégia que visa a apoiar, proteger e promover o aleitamento materno por meio da mobilização de profissionais de estabelecimentos de saúde. O objetivo dessa estratégia é fazer com que os profissionais reavaliem suas práticas e rotinas relativas ao aleitamento, muitas vezes responsáveis por alto índices de desmame precoce. Esta estratégia, criada em 1990 pelo Fundo das Nações Unidas para a Infância (UNICEF) e pela Organização Mundial da Saúde (OMS), possui como diretriz um conjunto de medidas denominadas "Dez Passos" para o sucesso do aleitamento materno, apresentados no Quadro 1.

Quadro 1 - Dez passos para o sucesso do aleitamento materno.

1. Ter uma norma escrita sobre aleitamento materno, rotineiramente transmitida a toda equipe de saúde.

2. Treinar toda a equipe de cuidados de saúde, capacitandoa para implementar esta norma.

3. Informar a todas as gestantes sobre as vantagens e o manejo do aleitamento materno.

4. Ajudar as mães a iniciar a amamentação na primeira hora após o nascimento.

5. Mostrar as mães como amamentar e como manter a lactação, mesmo se vierem a ser separadas de seus filhos.

6. Não dar ao recém-nascido nenhum outro alimento ou líquido além do leite materno, a não ser que haja indicação médica.

7. Praticar o alojamento conjunto - permitir que mãe e o bebê permaneçam juntos 24 horas por dia.

8. Encorajar o aleitamento materno sob livre demanda.

9. Não dar bicos artificiais ou chupetas às crianças amamentadas ao seio.

10. Encorajar a criação de grupos de apoio ao aleitamento materno, e encaminhar mães aos mesmos, por ocasião da alta hospitalar ou ambulatorial.

O Brasil foi escolhido entre 12 países para dar início à "Iniciativa Hospital Amigo da Criança". Assim, em 1992, o Ministério da Saúde apoiado pela UNICEF e Organização Pan - Americana da Saúde deu início ao processo em ação conjunta com o
Programa Nacional de Incentivo ao Aleitamento Materno (LAMOUNIER, 1998).

O Hospital Universitário Regional do Norte do Paraná (HURNP), em Londrina, entendendo a importância da proposta para aumentar as taxas de aleitamento materno das crianças nascidas nesta instituição, decidiu no início de 1995 desencadear o processo de implantação da IHAC.

Este trabalho tem como objetivos descrever o processo de implantação da IHAC no HURNP e seus resultados; relatar as dificuldades encontradas durante a implantação da IHAC no HURNP e salientar os benefícios resultantes da IHAC para o HURNP.

\section{Processo de Implantação da IHAC no HURNP}

O HURNP possui 294 leitos e é o único hospital público de grande porte de nível terciário do Norte do Paraná, o que o torna centro de referência regional no atendimento de todas as especialidades médicas. Pertence à Universidade Estadual de Londrina e está localizado no Centro de Ciências da Saúde, e isso o o caracteriza como um hospital-escola, que serve para a formação de alunos dos cursos de Enfermagem, Farmácia-Bioquímica, Fisioterapia e Medicina.

Por ser um hospital de referência regional, possui os 17 leitos de sua maternidade ocupados em sua maioria por gestantes de alto risco, encaminhadas dos ambulatórios de pré-natal, de consultórios e de outros hospitais de Londrina e região. Em 1974 foi implantado o alojamento conjunto e a partir de 1994 passou a contar com uma Unidade de Terapia Intensiva (UTI) com 7 leitos e uma Unidade de Cuidados Intermediários (UCI), com 10 leitos.

O trabalho de estímulo ao aleitamento materno no HURNP teve início na década de 70 por meio de inúmeras iniciativas que resultaram em aumentos significativos das taxas de aleitamento materno (FAVORETO; THOMSON, 1991; THOMSON, 1996; VANNUCHI et al., 1996). Apesar dos avanços durante quase três décadas, verificou-se a ne- 
cessidade de integrar ações no sentido de se obter melhores resultados. Esta oportunidade surgiu quando profissionais da área materno-infantil tomaram a decisão de reestruturar as rotinas relativas ao aleitamento materno e praticar os "Dez Passos" para o sucesso da amamentação preconizados pela IHAC.

Em 1995 dá-se início ao processo de implantação da IHAC no HURNP, conforme mostra o Quadro 2.

Quadro 2- Etapas desenvolvidas para a implantação da IHAC no HURNP.*

\begin{tabular}{|c|c|c|}
\hline \multicolumn{2}{|r|}{ ETAPAS } & DATAS \\
\hline 1. & $\begin{array}{l}\text { Diagnóstico da situação do HURNP com } \\
\text { relação aos Dez Passos da IHAC }\end{array}$ & março de 1995 \\
\hline 2. & $\begin{array}{l}\text { Formação do grupo de implantação da } \\
\text { IHAC no HURNP e início das atividades }\end{array}$ & abril de 1995 \\
\hline 3. & $\begin{array}{l}\text { Solicitação a Secretaria de Saúde do } \\
\text { Estado do Paraná (SESA) para pré- } \\
\text { avaliação dos Dez Passos }\end{array}$ & dezembro de 1997 \\
\hline 4. & Realização da pré-avaliação pela SESA & setembro de 1999 \\
\hline 5. & $\begin{array}{l}\text { Encaminhamento ao Ministério da Saúde } \\
\text { (MS) do resultado da pré- avaliação dos } \\
\text { Dez Passos, realizada pela SESA }\end{array}$ & setembro de 1999 \\
\hline 6. & $\begin{array}{l}\text { Avaliação dos Dez Passos da IHAC no } \\
\text { HURNP, pelo MS }\end{array}$ & abril de 2000 \\
\hline 7. & $\begin{array}{l}\text { Credenciamento do HURNP como } \\
\text { "Amigo da Criança, pelo MS }\end{array}$ & abril de 2000 \\
\hline 8. & $\begin{array}{l}\text { Entrega da placa de "Hospital Amigo da } \\
\text { Criança ao HURNP }\end{array}$ & setembro de 2000 \\
\hline
\end{tabular}

Fonte: Vannuchi (2002)

Durante o processo de implantação/implementação da IHAC no HURNP, dois inquéritos foram realizados com o objetivo de verificar o cumprimento dos "Dez Passos". O primeiro inquérito foi realizado no início de 1995, antes da implantação da IHAC (THOMSON et al., 1995), e o segundo no final de 1998 (SENTONE et al., 2000). Ambos os inquéritos foram realizados por profissionais de saúde integrantes do grupo de implantação da IHAC no HURNP, e tiveram como base o questionário de autoavaliação de hospitais fornecido pelo MS (BRASIL, 1992a). Para cada um dos "Dez Passos", foi definida uma forma de avaliação, incluindo a análise crítica das rotinas, o levantamento de prontuários de re- cém-nascidos (para verificar o tipo de alimentação recebida durante a internação) e a aplicação do questionário a gestantes, puérperas e profissionais de saúde do ambulatório de pré-natal, maternidade, UTI e UCI neonatal do HURNP.

O Quadro 3 apresenta um resumo dos resultados dos inquéritos realizados no HURNP, antes e após a implantação da IHAC.

Quadro 3 - Comparação do cumprimento dos "Dez Passos" pelo HURNP antes (1995)* e após (1998)** a implantação da IHAC.

\begin{tabular}{|c|c|c|c|}
\hline & \multirow[b]{2}{*}{ Passos } & \multicolumn{2}{|c|}{ Cumprimento dos Passos } \\
\hline & & 1995 & 1998 \\
\hline 1 & $\begin{array}{l}\text { Ter norma escrita sobre AM, que } \\
\text { deve ser rotineiramente transmitida } \\
\text { à equipe de saúde }\end{array}$ & Não & Sim \\
\hline 2 & $\begin{array}{l}\text { Treinar toda a equipe de cuidados } \\
\text { de saúde, capacitando-a para } \\
\text { implementar esta norma }\end{array}$ & $\begin{array}{c}\text { Parcial } \\
\text { (10,0\% da equipe) }\end{array}$ & $\begin{array}{l}\text { Parcial } \\
\text { (90,0\% da } \\
\text { equipe) }\end{array}$ \\
\hline 3 & $\begin{array}{l}\text { Informar todas as gestantes sobre } \\
\text { as vantagens e o manejo do } \\
\text { aleitamento }\end{array}$ & $\begin{array}{c}\text { Parcial } \\
(10,0 \% \text { das } \\
\text { gestantes) }\end{array}$ & $\begin{array}{c}\text { Total } \\
(100,0 \% \text { das } \\
\text { gestantes })\end{array}$ \\
\hline 4 & $\begin{array}{l}\text { Ajudar as mães a iniciar a } \\
\text { amamentação na primeira meia } \\
\text { hora após o parto }\end{array}$ & Não & Sim \\
\hline 5 & $\begin{array}{l}\text { Mostrar às mães como amamentar } \\
\text { e manter a lactação, mesmo se } \\
\text { vierem a ser separadas de seus } \\
\text { filhos }\end{array}$ & $\begin{array}{c}\text { Parcial } \\
(6,7 \% \text { das mães) }\end{array}$ & $\begin{array}{l}\text { Total } \\
(100,0 \% \text { das } \\
\text { mães })\end{array}$ \\
\hline 6 & $\begin{array}{l}\text { Não dar a recém-nascidos nenhum } \\
\text { outro alimento ou bebida além do } \\
\text { leite materno, a não ser que seja } \\
\text { indicado pelo médico }\end{array}$ & $\begin{array}{c}\text { Parcial } \\
(6,7 \% \text { dos RNs em } \\
\text { AME) }\end{array}$ & $\begin{array}{c}\text { Parcial } \\
\text { (80,2\% dos RNs } \\
\text { em AME) }\end{array}$ \\
\hline 7 & $\begin{array}{l}\text { Praticar o alojamento conjunto - } \\
\text { permitir que mães e bebês } \\
\text { permaneçam juntos } 24 \mathrm{~h} \text { por dia }\end{array}$ & $\begin{array}{l}\text { Parcial } \\
(50,0 \% \text { - Parto } \\
\text { Normal })\end{array}$ & $\begin{array}{l}\text { Total } \\
(100,0 \% \text { dos } \\
\text { RNs })\end{array}$ \\
\hline & & (100,0\% - Cesária) & \\
\hline 8 & $\begin{array}{l}\text { Encorajar o aleitamento sob livre } \\
\text { demanda }\end{array}$ & $\begin{array}{c}\text { Parcial } \\
\text { (6,7\% das mães) }\end{array}$ & $\begin{array}{l}\text { Total } \\
(100,0 \% \text { das } \\
\text { mães })\end{array}$ \\
\hline 9 & $\begin{array}{l}\text { Não dar bicos ou chupetas a } \\
\text { crianças amamentadas ao seio }\end{array}$ & $* * *$ & $\begin{array}{c}\text { Total } \\
(100,0 \% \text { dos } \\
\text { RNs })\end{array}$ \\
\hline 10 & $\begin{array}{l}\text { Encorajar a formação de grupos de } \\
\text { apoio à amamentação para onde as } \\
\text { mães devem ser encaminhadas, } \\
\text { logo após a alta do hospital ou } \\
\text { ambulatório**** }\end{array}$ & $\begin{array}{l}\text { Parcial } \\
\text { (esporádico ao } \\
\text { BLH) }\end{array}$ & $\begin{array}{l}\text { Total } \\
(100,0 \% \text { das } \\
\text { mães })\end{array}$ \\
\hline
\end{tabular}

Fontes:

* Thomson, Vannuchi e Melanda, 1995.

** Sentone, 2000.

*** A avaliação deste passo ficou prejudicada em função do período de transição para a rotina do uso do "copinho".

**** Não existem grupos de apoio comunitários em Londrina. O cumprimento do passo foi avaliado considerando-se o encaminhamento das mães aos serviços de apoio do HURNP. 
Para o Passo 1 no início de 1995, de acordo com resultados do primeiro inquérito, não havia norma específica sobre aleitamento materno na UTI/UCI neonatal do HURNP. O que existia era um material bastante volumoso intitulado "Normas, TÉCNICAS E Rotinas do Setor de Enfermagem da Unidade de NeONAtologia”, o qual continha todas as normas, técnicas e rotinas para a organização das atividades desenvolvidas no setor, dirigido aos três turnos de trabalho. A técnica de Aleitamento Materno (AM) encontrava-se descrita junto às demais, sem realce para tal procedimento.

Após a IHAC foram implantadas normas e rotinas específicas sobre AM na UTI e UCI neonatal, sala de parto e maternidade conforme resultado demonstrado no inquérito de 1998.

Quanto ao treinamento da equipe (Passo 2), o inquérito realizado em 1995 revelou que apenas $10,0 \%$ dos funcionários haviam recebido treinamento específico sobre AM. Já em 1998, 90,0\% da equipe havia sido treinada, porém, com relação à UTI/ UCI neonatal, toda a equipe havia recebido treinamento. O processo de treinamento coletivo foi desencadeado somente após a IHAC.

O inquérito realizado em 1995 mostrou que as orientações dadas às mães no pré-natal (Passo 3), eram insuficientes e atingiam um número muito pequeno de mães $(10,0 \%)$. Já o segundo inquérito revelou que todas as mães recebiam orientações no pré-natal.

Com relação ao passo 4 , o primeiro inquérito mostrou que, no início de 1995, este passo não era obedecido. Nos casos de parto normal, apenas uma criança havia sido amamentada com menos de três horas após o nascimento, enquanto 70,0\% haviam sido amamentadas com mais de seis horas de vida. Nos casos de cesariana, todas as crianças haviam sido amamentadas com mais de oito horas de vida. Apenas 20,0\% das mães disseram ter recebido orientações sobre o início da amamentação. O inquérito de 1998 revelou que todas as mães e recém-nascidos em boas condições de vitalidade, haviam recebido ajuda para iniciar a amamentação na primeira meia hora de vida.
No primeiro inquérito, 93,3\% das mães informaram não ter recebido as orientações contidas no Passo 5, quando separadas de seus filhos. Por outro lado, no segundo inquérito, todas as mães separadas de seus filhos disseram ter recebido essas orientações.

O primeiro inquérito evidenciou o descumprimento total do Passo 6, pois, de acordo com a maioria dos prontuários médicos, o leite artificial foi ministrado sem prescrição médica e raras foram as justificativas encontradas nos prontuários para não usar o leite materno. Somente $6,7 \%$ das crianças encontravam-se em Aleitamento Materno Exclusivo (AME). Em 1998, essa situação se altera, e todos os casos de crianças que receberam outro alimento ou líquido diferente do leite materno foram justificados, conforme anotação constante em prontuário. Das crianças pesquisadas no segundo inquérito, $80,2 \%$ encontravam-se em AME.

Para o passo 7, o primeiro inquérito revelou que, até o início de 1995, todos os recém-nascidos de parto cirúrgico, em boas condições de vitalidade, eram alojados após 12 horas de vida, e 50,0\% dos recém-nascidos de parto normal eram alojados a partir de três horas de vida. Após a IHAC, de acordo com o segundo inquérito, todos os recém-nascidos de parto normal ou cirúrgico em boas condições de vitalidade, eram alojados imediatamente após o nascimento.

O inquérito de 1995 mostrou, para o Passo 8, que a maioria das mães $(93,3 \%)$ não haviam recebido informações acerca da frequiência das mamadas; já o inquérito de 1998 revelou que todas as mães afirmaram ter recebido orientação nesse sentido.

$\mathrm{Na}$ época do primeiro inquérito, a avaliação do Passo 9 ficou prejudicada porque estava ocorrendo na UTI/UCI neonatal e na maternidade do HURNP, a substituição de bicos artificiais pelo "copinho" para oferecimento de leite aos bebês. Dentre as crianças amamentadas com leite artificial (93,3\%), algumas já o haviam recebido pelo "copinho". Com relação ao uso de chupeta, todas as mães afirmaram que seus filhos não a usavam. As crianças em fototerapia recebiam, rotineiramente, chás em mamadeiras. Após a IHAC, houve abolição desta prática, como demonstrou o segundo inquérito. 
Para o Passo 10, o primeiro inquérito mostrou que o serviço de apoio ao AM mantido pelo HURNP era o BLH, porém, a rotina existente naquela época, não era clara quanto ao encaminhamento das mães a esse serviço no momento da alta hospitalar. Nenhuma mãe referiu o encaminhamento a grupos comunitários de apoio ao AM, somente Postos de Saúde e ambulatórios do próprio HURNP foram citados. O inquérito de 1998 verificou que todos os recémnascidos, de acordo com a necessidade, são agendados previamente para os serviços de apoio ao AM do HURNP - Banco de Leite Humano, Clínica de Lactação, Ambulatório de Puericultura e Ambulatório de Prematuridade, uma vez que não há, em Londrina, grupos de apoio ao AM organizados pela comunidade.

\section{Dificuldades na Implantação da IHAC no HURNP}

Por se tratar de um hospital-escola, algumas decisões devem ser tomadas em comum acordo entre as direções do HURNP e do Centro de Ciências da Saúde (CCS), o qual congrega os docentes responsáveis pelos alunos que desenvolvem atividades acadêmicas no hospital. O descompasso entre os níveis de decisão gera morosidade, tornando-se um complicador para se iniciar o processo na prática.

No início do processo de implantação existiu uma forte adesão por parte da maioria de docentes do setor materno-infantil, porém algumas resistências foram manifestadas, dificultando e postergando o desencadeamento do processo. Uma vez tomada a decisão de se implantar a IHAC, foi criado um grupo composto por enfermeiros, médicos, fisioterapeuta, nutricionista e assistente social, responsável por desenvolver estratégias voltadas à implantação e implementação da IHAC no HURNP.

Este grupo a priori era numeroso, mas, com o passar do tempo, ficou restrito a poucas pessoas, principalmente àquelas mais comprometidas com o processo de mudança, resultando em mais uma dificuldade dada a sobrecarga de trabalho acarretada aos profissionais que permaneceram no grupo.
A exemplo de outros hospitais-escola, o HURNP convive com uma rotatividade muito grande de alunos, o que dificulta a implantação do Passo 2, que trata do treinamento de toda a equipe para implementar as normas e rotinas relativas ao aleitamento materno. Desde a implantação da IHAC, foram realizados 26 cursos, perfazendo um total de aproximadamente 500 pessoas treinadas, necessitando assim de pelo menos um curso de treinamento a cada seis meses. Mesmo assim, ainda não é possível o treinamento de todos os alunos dos cursos da área de saúde que atuam nas unidades materno-infantil, procurando-se oferecer o treinamento aos alunos e residentes do curso de medicina, devido ao fato de serem os que permanecem maior tempo nas unidades em foco.

Mudar rotinas que se perpetuaram por anos e paradigmas existentes, não se apresentaram como tarefas de fácil realização. Dificuldade de aceitação da mamada na sala de parto por parte dos obstetras e anestesistas, foi um desafio significativo a se transpor. Batalhas, literalmente, foram travadas a fim de se efetivar esta rotina. O número reduzido de funcionários apresentou-se como uma dificuldade, visto que foram necessárias mudanças no processo de trabalho.

A substituição das chucas por copinhos para administração de leite aos recém-nascidos (RN) não representou grande dificuldade, porque já havia sido instalado o processo de substituição antes de se desencadear a IHAC no HURNP. Entretanto surgiu a dificuldade, que persiste até o momento, que é o controle exato do volume de leite oferecido a criança. Durante a administração por copo há perda de volume, o que gera insegurança por parte do funcionário que administra leite ao $\mathrm{RN}$, principalmente quando a criança continua chorando após a alimentação.

A maioria dos funcionários das unidades de neonatologia e maternidade são lotados há muito tempo nestes setores desenvolvendo práticas que muitas vezes não favoreciam o aleitamento materno. Isso no início dificultou as mudanças de normas e rotinas 
que foram estabelecidas, como por exemplo a cultura da chupeta e bicos, dar de mamar de $3 \mathrm{em} 3$ horas etc., foram desafios transpostos no dia-a-dia.

A integração entre as unidades de neonatologia e Banco de Leite Humano (BLH) já existia, porém houve necessidade de reestruturação do fluxo de leite humano proveniente do banco e distribuído pelo lactário afim de otimizar a chegada deste leite ao RN.

\section{Benefícios Resultantes da Implantação da IHAC no HURNP}

Muitos foram os benefícios da implantação da IHAC no HURNP, entre eles salientamos o aumento do índice de aleitamento materno exclusivo, atualmente em torno de $95 \%$. Este fato é decorrente de uma somatória de esforços de toda a equipe que mudou comportamentos e assumiu atitudes de promoção, proteção e apoio ao aleitamento materno.

A melhora do entrosamento e da integração entre os diversos setores envolvidos com a IHAC foi um fator que proporcionou harmonia às equipes.

Os cursos sobre Manejo e Promoção do Aleitamento materno possibilitaram um melhora na capacitação das equipes, instrumentalizando-as para mudanças de atitudes bem como proporcionando uma reflexão crítica da prática de cada um.

Mudanças no processo de cuidar do recém-nascido e mãe foi um passo desafiador, porém, gratificante, uma vez que se colocou em prática a assistência de enfermagem integral ao binômio mãe e filho, tornando a assistência mais humanizada.

A criatividade e o envolvimento das pessoas em desenvolver e sistematizar estratégias de divulgação do aleitamento materno culminaram na elaboração da cartilha "DICAS PARA O SUCESSO DA AMAMENTAÇÃO, folder "COMO AJUDAR AS MÃES A AMAMENTAR", elaboração de um kit para as puérperas sobre aleitamento materno, posters e trabalhos científicos.
Uma maior monitorização das dificuldades das mães em relação ao manejo do aleitamento materno foi capaz de diminuir o estresse da mãe e do recémnascido, o número de fissuras, ingurgitamentos entre outros e possibilitou um reconhecimento das mães do trabalho que está sendo desenvolvido.

\section{Considerações Finais}

A implantação da IHAC no HURNP, hospitalescola e com características próprias reverteu-se em um grande desafio.

Foram cinco anos de caminhada até a conquista do título de Hospital Amigo da Criança, o que representou mudanças de comportamento e práticas, que culminaram no aumento dos índices de aleitamento materno exclusivo e a participação da equipe no contexto de estratégias nacionais para diminuir os índices de mortalidade infantil em nosso país.

Ressaltamos a necessidade, por ser um hospitalescola, de que o conteúdo do curso sobre "Manejo e Promoção do Aleitamento Materno" seja inserido na grade curricular dos cursos da área da saúde. Assim, é facilitada a inserção dos alunos no contexto de um Hospital Amigo da Criança, aliviando a tarefa de realização de vários cursos de treinamento num mesmo ano.

Acreditamos que a partir do momento em que as pessoas são levadas a refletir sobre o seu trabalho e o significado que este possui, a probabilidade de mudanças pessoais é grande, repercutindo em transformações na sociedade e em conquistas de muitos desafios.

\section{Referências}

FAVORETO, J., THOMSON, Z. Avaliação do programa de estímulo ao Aleitamento materno do Hospital Universitário do Norte do Paraná, Londrina. J. Pediatr., Rio de Janeiro, v. 70, p. 138-49, 1991.

LAMOUNIER, J. A. Experiência iniciativa Hospital Amigo da Criança. Rev. Ass. Med. Brasil, São Paulo,v. 44, n. 4, p. 319-24, 1998. 
SENTONE, A. D. D. et al. Caminhos de uma conquista. Londrina, 2000. Painel apresentado por ocasião da entrega da placa ao HURNP de "Hospital Amigo da Criança".

THOMSON Z; VANNUCHI M.T.O.; MELANDA M.A. Relatório sobre a situação do HURNP em relação à Iniciativa Hospital Amigo da Criança. Londrina, 1995. Material digitado e não publicado.

THOMSON, Z. (coord.). Projeto Centro de Referência em aleitamento materno em Londrina. Londrina: Universidade Estadual de Londrina, 1996.
VANNUCHI, M. T. O. Avaliação da Iniciativa Hospital Amigo da Criança na Unidade de Neonatologia do Hospital Universtário Regional do Norte do Paraná, Londrina. São Paulo, 2002. Tese (Doutorado) - Faculdade de Saúde Pública da USP.

VANNUCHI, M. T. O. et al. Banco de Leite do Hospital Universitário Regional do Norte do Paraná: Unindo esforços no incentivo ao aleitamento materno. In: MOSTRA DE EXPERIÊNCIAS DO SERVIÇO MUNICIPAL DE SAÚDE DE LONDRINA, 1. Londrina. Anais... Londrina:[s.n], 1996. 
